\title{
ABSTRACT
}

DOI: $10.1002 /$ zaac.200((will be filled in by the editorial staff)

\section{Valence changes in dicarbide solid solutions induced by structural changes Uwe Ruschewitz, ${ }^{*[a]}$ Stefanie Busch ${ }^{[a]}$}

* Prof. Dr. U. Ruschewitz

E-Mail: uwe.ruschewitz@uni-koeln.de

[a] Department für Chemie

Universität zu Koeln

Greinstr. 6

50939 Köln

Keywords: solid solution, valence change, carbide

The rare-earth metal carbides $\left(\mathrm{REC}_{2}\right) \mathrm{EuC}_{2}$ and $\mathrm{YbC}_{2}$ are isostructural to alkaline-earth metal carbides $\left(\mathrm{AEC}_{2}\right)$, both containing $\mathrm{C}_{2}$ dumbbells. Despite their similar crystal structures completely different physical properties are observed, i.e. metallic $\left(\mathrm{REC}_{2}\right)$ versus insulating $\left(\mathrm{AEC}_{2}\right)$ behaviour. In this context, semiconducting properties with divalent $\mathrm{Eu}(\mathrm{II})$ under ambient conditions are found for $\mathrm{EuC}_{2}{ }^{[1]}$, but under pressure metallic $\mathrm{EuC}_{2}$ with trivalent $\mathrm{Eu}(\mathrm{III})$ is observed. ${ }^{[2]}$ However, for $\mathrm{YbC}_{2}$ an intermediate valence of approx. 2.8 was determined. ${ }^{[3]}$ As confirmed by XRPD data at RT we were able to synthesize several solid solutions by reaction of the elements, e.g. $\mathrm{Yb}_{\mathrm{x}} \mathrm{Sr}_{1-\mathrm{x}} \mathrm{C}_{2}{ }^{[4]}, \mathrm{Eu}_{\mathrm{x}} \mathrm{Ca}_{1-\mathrm{x}} \mathrm{C}_{2}$, $\mathrm{Eu}_{\mathrm{x}} \mathrm{Ba}_{1-\mathrm{x}} \mathrm{C}_{2}$ and $\mathrm{Yb}_{\mathrm{x}} \mathrm{Ca}_{1-\mathrm{x}} \mathrm{C}_{2}{ }^{[4]}(0<\mathrm{x}<1)$. As a result, all solid solutions crystallize in different modifications depending on $\mathrm{x}$. In addition, solid solutions containing Eu show a clear tendency towards a perfect vegard behaviour as reported for $\mathrm{Eu}_{\mathrm{x}} \mathrm{Sr}_{1-\mathrm{x}} \mathrm{C}_{2}{ }^{[5]}$, whereas solutions containing $\mathrm{Yb}$ show unusual effects that point to a change of $\mathrm{Yb}$ valence stimulated by composition, temperature and different crystal structures. .

[1] D. Wandner et al., Inorg. Chem. 2010, 49, 312.

[2] U. Ruschewitz, K. Syssen et al., unpublished results.

[3] a) M. Atoji, J. Chem. Phys. 1961, 35, 1950; b) P. Link et al. Inorg. Chem. 2011, 50, 5587.

[4] P. Link, Doktorarbeit, Köln, 2011.

[5] P. Link et al., Z. Anorg. Allg. Chem. 2010, 636, 2276. 